\title{
Research Paper: Comparing the Effect of Core Stability Exercises and Electrotherapy on Nonspecific Chronic Low crossuark Back Pain in Mother Assistants Working in Mentally or Physical Retarded Children Wards
}

\author{
Nasrin Fouladi ${ }^{1}$, Behnaz Ganji ${ }^{*}$, Yahya Sokhangoei ${ }^{3}$
}

1. MSc., Department of Corrective Exercise, Faculty of Physical Education and Sport Sciences, Karaj Branch, Islamic Azad University, Karaj, Iran. 2. Assistant Professor, Department of Sport Injuries and Corrective Exercises, College of Physical Education, Karaj Branch, Islamic Azad University, Karaj, Iran 3. Assistant Professor, Department of Rehabilitation Sciences, University of Social Welfare and Rehabilitation Sciences, Tehran, Iran.

\begin{tabular}{|c|c|}
\hline $\begin{array}{l}\text { Use your device to scan } \\
\text { and read the article online }\end{array}$ & $\begin{array}{l}\text { Citation: Fouladi N, Ganji B, Sokhangoei Y. Comparing the Effect of Core Stability Exercises and Electrotherapy on Non- } \\
\text { specific Chronic Low Back Pain in Mother Assistants Working in Mentally or Physical Retarded Children Wards. Journal of } \\
\text { Modern Rehabilitation. 2017; 11(1):63-72. https://doi.org/10.18869/nirp.jmr.11.1.63 }\end{array}$ \\
\hline a & dol': https://doi.org/10.18869/nirp.jmr.11.1.63 \\
\hline
\end{tabular}

Article info:

Received: 12 Sep. 2016

Accepted: 23 Nov. 2016
Keywords:

Core stability exercises, Electrotherapy, Chronic nonspecific low back pain

\section{ABSTRACT}

Introduction: Low Back Pain (LBP) is the most common cause of daily activities restriction and functional disability. Of various chronic LBPs, the most common type is chronic non-specific LBP. This study aimed to compare the effect of core stability exercises and electrotherapy on relieving symptoms of chronic non-specific LBP in mother assistants working in caring centers for mentalphysical disabled children.

Materials and Methods: A total of 42 mother assistants with nonspecific chronic LBP were randomly selected and put into three groups: the stabilization exercises $(n=15)$, the electrotherapy $(n=15)$, and the control $(n=12)$. Their mean(SD) age was 40.3(1.8) years; mean(SD) work background: $15.8(7.6)$ years, mean(SD) weight: $69(11.8) \mathrm{kg}$, and mean(SD) height: $161.5(6.3) \mathrm{cm}$. The first group underwent a 6-week training of core stability exercises every other day; the second group received a 10-session electrotherapy; however, the third group received no treatment at all. The three groups were given pretests and posttests via completing McGill Pain Questionnaire at the of the study.

The 1-way analysis of variance to test all variables showed that all groups were matched in terms of job background, height, weight and age. The Shapiro-Wilk test results showed that the difference between the scores of pretest and posttest were normally distributed. To verify the hypotheses, ANOVA test was applied. All analyses were done at significance level of 0.05 using SPSS 22 .

Results: Results revealed a significant difference in the mean changes chronic LBP in working mother assistants in the electrotherapy group $(-31.5)$, and control group $(-1.7)(\mathrm{P}=0.000)$. In other words, a course of electrotherapy led to a significant reduction in chronic non-specific LBP in mother assistants.

Conclusion: Electrotherapy compared to core stability exercises contributes significantly in reducing nonspecific chronic LBP in mother assistants working in units for caring children with physical or mental disabilities.

* Corresponding Author: 


\section{Introduction}

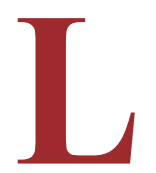

ow Back Pain (LBP) is the most common cause of daily activities restriction and functional disability in people under 45 years. It can incur huge costs directly and indirectly on individuals and society [1]. Chronic LBP is when the pain and disability lasts more than 3 months [2]. Of various chronic LBPs, the most common type is chronic non-specific LBP affecting 90\% of the patients [3]. Although no pathology has not been found for this type of pain, mechanical factors such as shortening and reducing muscular endurance are accounted in its etiology [4]. According to motor dysfunction syndrome and lack of muscle power, movement disorders, such as decreased flexibility or muscle strength can cause adverse changes in soft tissue and skeletal structure that may end in pain and functional limitations [5]. Thus, to plan a precise treatment for back pain, not only reducing pain but also correcting all mechanical factors that have cluttered the movement patterns should be targeted [6].

Activities requiring frequent changes in posture, lifting heavy loads, bending frequent rotations, can contribute to the development of LBP by unbalancing pressure exerted on the waist [7]. Therefore, mother assistants working in care centers endure much physical pressure on their lumbar spines, necks, and knees, for they have to remove disabled children who, because of their inactivity, may be overweight. Such mother assistants have to maintain a position of semi-curved back for long hours per day for dressing, feeding and bathing the handicapped children. Therefore, they often complain of calf and thigh cramps.

Some evidence indicates that people with chronic LBP and sacroiliac pain lack the efficient power in their core muscles and have a central body weakness. High prevalence of LBP and related disabilities and impairments in carrying out daily activities will have a very negative impact on patients and society. There are different types of back pain. Based on the etiology, they are divided into two categories: specific or pathological and non-specific or mechanical [8]. The specific type is due to factors such as stenosis and pressure on nerve roots caused by tumors or venous congestion or infection [9]. In non-specific types, no special pathology is involved, such as muscle spasms, disorders of the spine and sciatica arc, etc [9].

The mechanical stability of the spine, especially in moving and heavy load-bearing tasks is maintained by spine and surrounding muscles. The spine stabilization system was first proposed by Punjabi in 1992. This system consists of three parts: 1 . the spine providing inher- ent stability [10]; 2. The muscles surrounding the spine providing dynamic stability and 3 . Neural control unit providing muscle response. In a normal situation these three systems coordinate their duties to provide mechanical stability. Different parts of the spine send information about the status of its mechanical situation such as location, load and motion of each vertebrae in dynamic mode. Neural control unit processes this information to provide the stability needed to produce the appropriate muscle movement patterns [11]. Costa et al. [12] and Mottalebi Lina et al. [13] concluded that core stability exercises increase muscle cross-sectional area of multifidus and thus reduce the pain.

A large number of acute and chronic musculoskeletal syndromes like chronic LBP are because of the accumulation of micro trauma caused by stress. These stresses are caused by either repetitive movements in a particular direction or keeping directions going in an unfavorable situation [14]. Electrical stimulation is widely used as an alternative medicine to control pain. The use of this treatment plan is based on pain gate theory. Another use of electrical stimulation is the placebo effect used to relieve pain while performing electrical stimulation of modalities [15]. Research performed on the effects of electrotherapy on patients with nonspecific chronic LBP indicate that these methods reduces the pain [16].

\section{Materials and Methods}

This study was approved by the Management Committee of Welfare Organization. The study objectives were fully explained to mother assistants and they announced their consent. They had the right to leave the study any time they wished. The present research is an applied and quasi-experimental study conducted with pretest and a posttest design. It is a comparative and impact assessment study. The research subjects were selected out of all mother assistants working in the mental health care centers for children with disabilities in Narmak and Rofideh welfare centers of Tehran. The study sample comprised 42 mother assistants and were randomly divided into three groups of core stability training, electrotherapy and control. Their mean(SD) age was 40.3(1.8) years; mean(SD) work background: 15.8(7.6) years, mean(SD) weight: 69(11.8) $\mathrm{kg}$, and mean(SD) height: $161.5(6.3) \mathrm{cm}$.

Demographic characteristics of the participants and study variables were presented by descriptive statistics such as mean and standard deviation. The normal distribution assumption of the data was checked by ShapiroWilk test. The study hypotheses were tested using 1-way 
analysis of variance. All analyses were performed at the significance level of 0.05 using SPSS 22.

The inclusion criteria included having chronic nonspecific LBP with or without foot infection, and suffering from the pain for at least three months with no improvement. However those who had not gotten improved after resting and their pain would return in case of having activity were forbidden from exercising. These mother assistants were selected according to their referral from specialists in neurology or orthopedics. Exclusion criteria were having infection, tumors, rheumatoid disease, vertebral fracture, spondylolisthesis, pain; and urinary tract infections in women; under analgesic and anti-inflammatory drugs medication and son on [17]. The mother assistants' medical history and information such as date of LBP onset, duration of current back pain, age, height, weight, and work experience were recorded.

\section{Data collection instruments}

McGill pain questionnaire was used to collect data [18]. Its validity has already been approved in Dworkin investigation [19]. The reliability was calculated using the Cronbach $\alpha$ coefficient that ranged between 0.83 and 0.87 for all dimensions (The subjects were given the necessary explanation on how to fill out the questionnaire). Then all participants completed consent forms to participate in the study. The subjects reported their pain perception based on 20-item questionnaire. In other words, in any given set, there were different options for describing the pain of which they would choose only one. The independent variable were core stability training and electrotherapy and the dependent variable was non-specific back pain.

\section{First group: Stabilizing exercises}

Fifteen assistants were placed randomly in this group. First, the participants were given a pretest. Then they were trained the proper implementation of all exercises during the session. Because some mother assistants with chronic back pain were afraid of doing the exercises, extra time and training were considered for them. Exercises were done every other day for a period of 6 weeks and their execution time were adjusted with the shift of mother assistants. Each session lasted for 30 to 45 minutes. Before doing the main exercises, a 10-minute warm-up including stretching exercises and walking fast was performed. At the end of each session there was cool down for 10 minutes [20]. Each exercise was repeated in three sets. Extra time and the gradual increase of each exercise were marked and controlled based on the proper implementation and the pressure of each ex- ercise in the previous session. The goal was to gradually increase the number of exercises for higher endurance. In the case of the cramps, the target was to increase the initial contraction time to 3 times. Each workout time was 3 seconds at the start and increased to 9 seconds in the last session. The number of exercises began with a 3 -item set and ended in three 3-item sets. The emphasis was on transverse abdomens and multifidus muscles. The exercises were performed progressively from nonweight bearing position to weight bearing position. In this group, the static and dynamic stabilization of the spine were applied [21].

After administering abdominal muscle activation techniques and transverse abdominal muscle was stimulation, the exercises were practiced in combination that included abdominal and pelvic muscle contraction and bridge press, four-handed situation of nail enhancing the feet of opposition, lying supine and abdominal muscle contraction and then bridging and simultaneously rising the pelvis [22]. Diaphragmatic breathing should be done with a natural rhythm, and whenever the patient achieved control on core stability exercises, she can progress to exercises using Physio Ball [22]. The mother assistants were given a posttest at the end of 6 weeks of performing core stability exercises. The researcher had a full control over the mother assistants and correct performance during the exercise.

\section{The first group: The stabilization exercises}

As shown in Figure 1 muscles targeted included transverse abdominal muscles and internal oblique. The objective was increasing the static stability, increasing endurance, and muscle strength of the lower abdomen. In Figure 2 the muscles targeted were all abdominal wall muscles The objective was increasing the static stability, the endurance power. As in Figure 3 the target muscles were the muscles of the abdominal wall and lumbar area. The objective was to increase and improve the level of preparedness of the lumbar spine and lumbopelvic complex.

As is shown in Figure 4 the targeted muscles included paraspinal muscles. The objective was increasing movement in the spine and strengthen the paraspinal muscles. As in Figure 5, muscles involved included extensor of the pelvis, abdominal muscles, and lumbar muscles. The objective was increasing dynamic stability and enhancing strength, increasing lumbar stability, strengthening hip extensor muscles.

As shown in Figure 6, the muscles involved were the extensor muscles of the lower back, hip and shoulder. The objective was increasing dynamic stability, muscle 


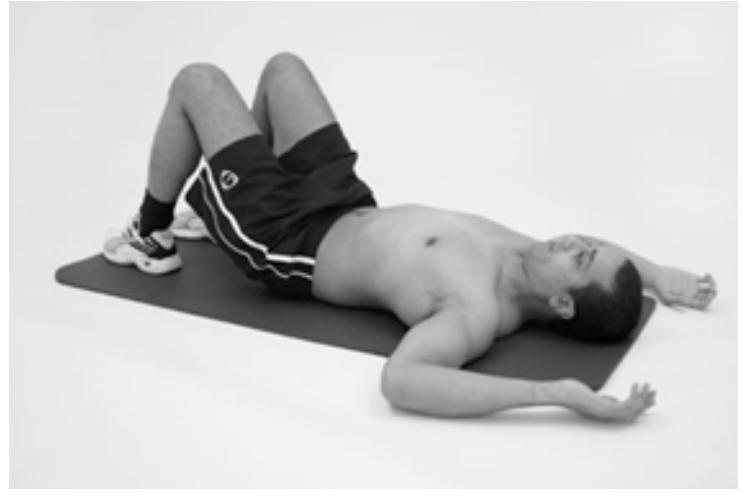

(a)

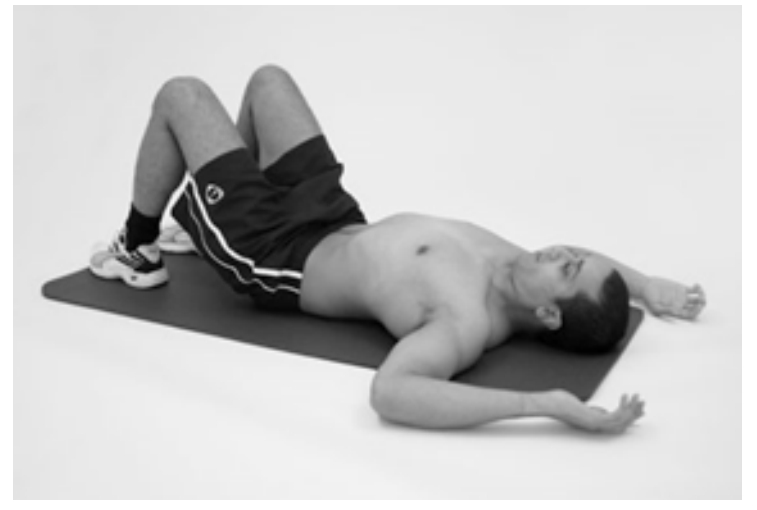

(b)

$J \mathrm{MR}$

Figure 1. First exercise: Belly deepening

strength. As in Figure 7, muscles involved were groups of the upper abdomen muscles. Its objective was increasing dynamic stability and strength of the muscles. As shown in Figure 8, target muscles included the muscles of the lower abdomen and lower back. The objective was increasing dynamic stability and strength of the muscles.

\section{The second group: Electrotherapy}

Fifteen participants were randomly selected and placed in this group. First, the participants were passed a pretest. Then the mother assistants received electrotherapy for 10 sessions every other day. Treatment included IR (15 $\mathrm{min})$, US continuously with a frequency of $1 \mathrm{MHz}$, intensity of 0.8 up to $3 \mathrm{~W} / \mathrm{cm}^{2}$ and 5 to 10 minutes TENS (20 minutes) or intermittent Interference (IF) (20 minutes). Because the effect was the same with both TENS and IF, only one of them was used in electrotherapy. The time of each session was 40 to 45 minutes and the style of putting electrodes was accorded with the place of pain [15]. In a study done on the effects of TENS and IF, it was concluded that both equally reduce pain and there was no difference between their effects.

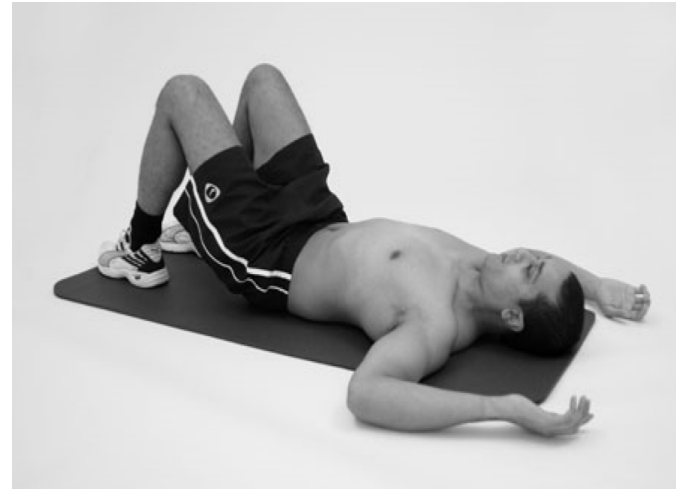

(a)

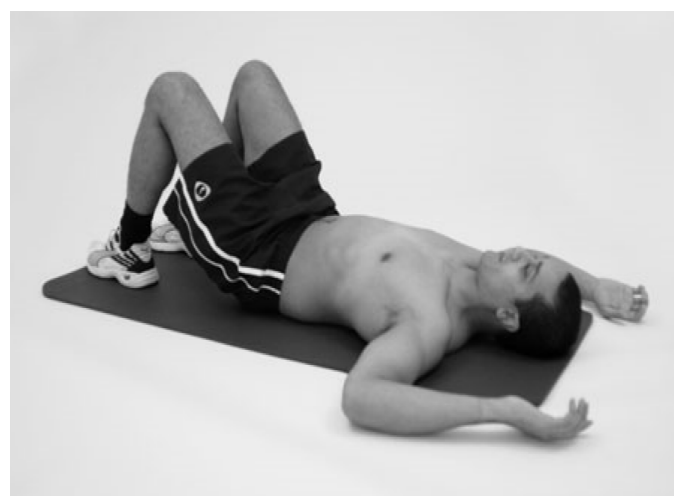

(b)

JMR

Figure 2. Second exercise: Tightening the abdominal cavity

At the beginning of each electrotherapy session, in order to set modalities, the patient was asked the current amount of pain compared to the previous session, and it was recorded in the patient's profile. The square pulses were used for electrical stimulation. According to the subjects' pain or relief level, the amplitude used in the study ranged between 10 and $100 \mathrm{~mA}$. The type of current used was alternative to be controlled by nerve stimulations. The medium frequency used ranged between 80 and $100 \mathrm{~Hz}$ based on the mother assistant's tolerance. Depending on the severity of pain and the patient's tolerance, the treatment time was 15 to 20 minutes. During

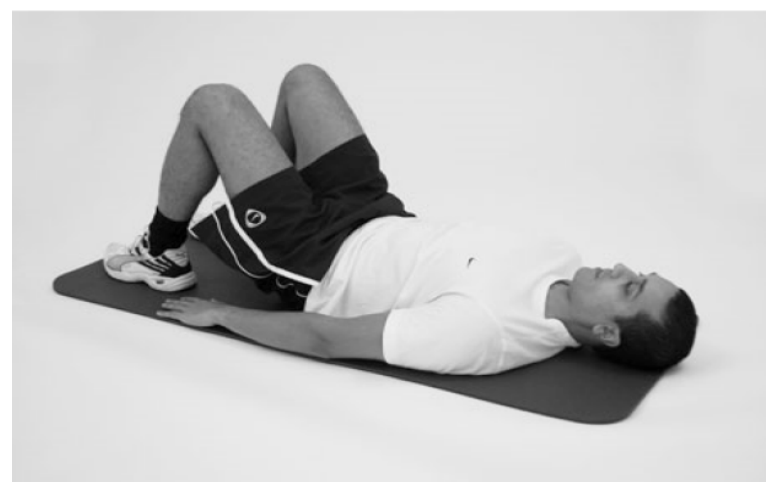

Figure 3. Third exercise: Pelvic tilt 


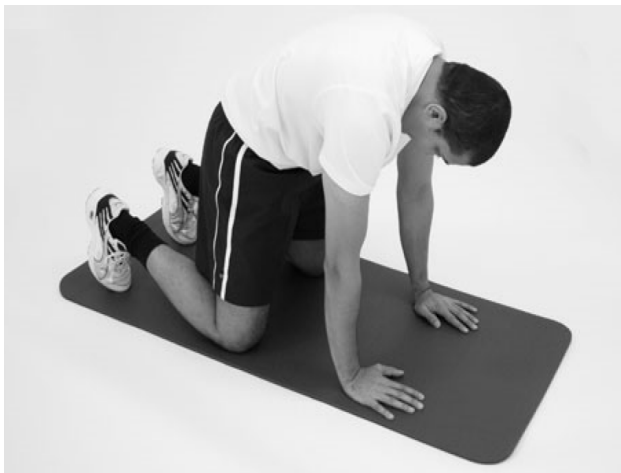

(a)

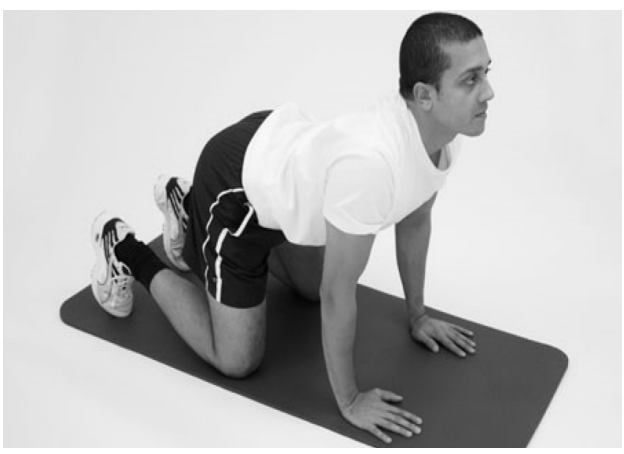

(b)

Figure 4. Fourth exercise: Cat-camel

$J \mathrm{MR}$

IF, the square pulse-type, alternative frequency and a frequency of $4 \mathrm{kHz}$ were applied.

The third group

This group was the control group and no core stability exercise or electrotherapy was performed on them. However, they were given a pretest at the beginning and a posttest at the end of the sixth week.

(a)

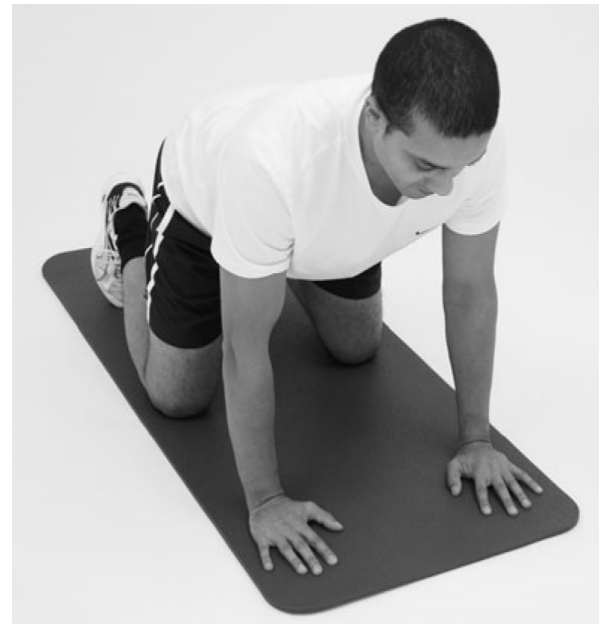

(b)

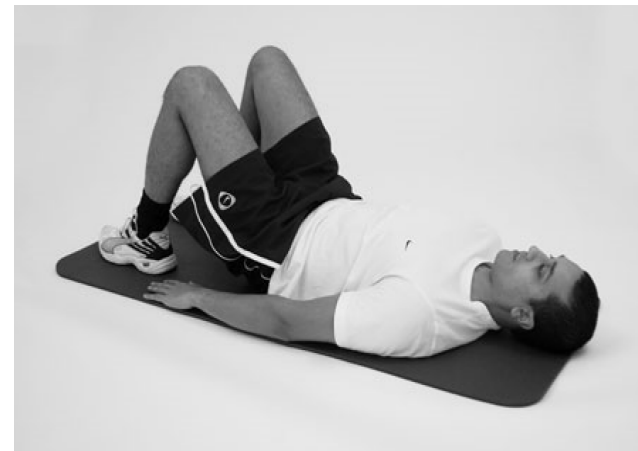

(a)

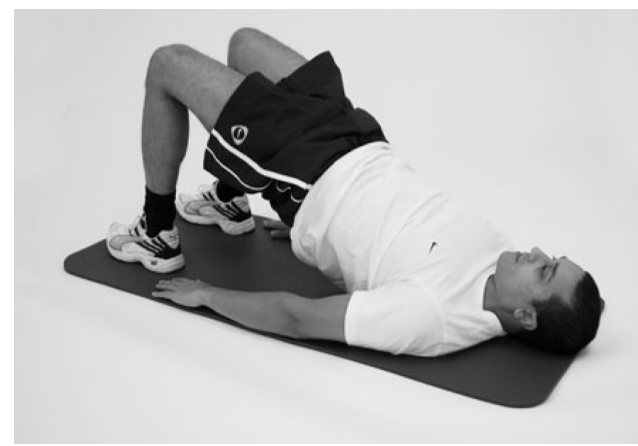

(b)

Figure 5. Fifth exercise: Bridging

$J \mathrm{MR}$

Statistical methods

The demographic characteristics of the participants and the research variables were described using descriptive statistics such as mean, standard deviation and the related tables and graphs. Since the pretest and posttest scores were normally distributed and the measuring scale was interval, variable is the distance. Therefore, in order to verify the research hypothesis, 1-way ANOVA was used. Shapiro-Wilk test was performed to verify normal

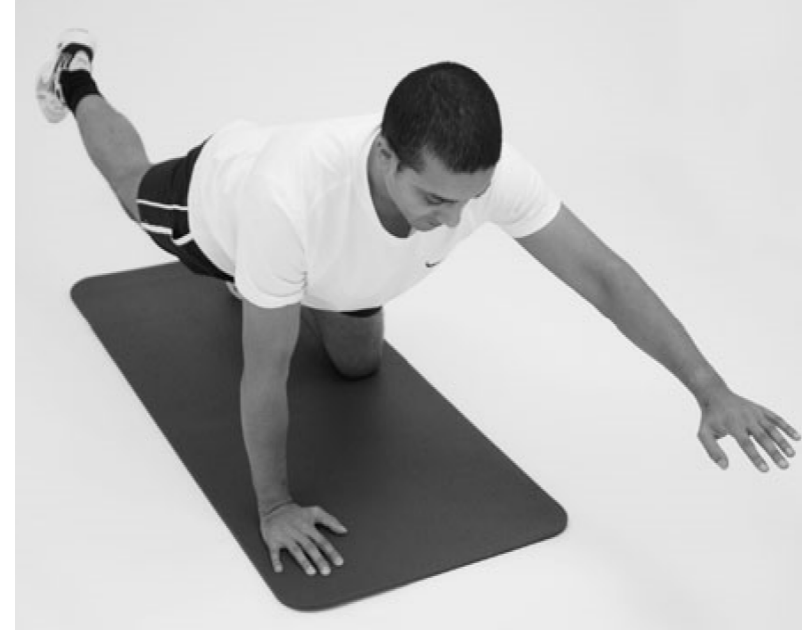

Figure 6. Sixth exercise: Four nail to stretch opposite arm and leg 


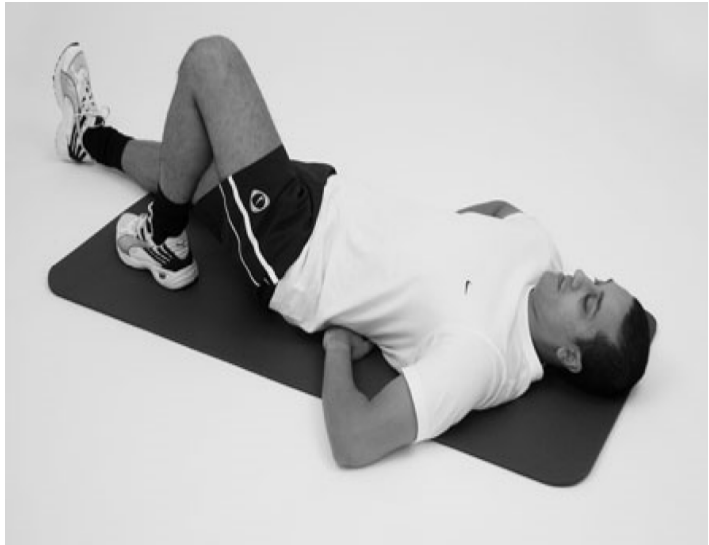

(a)

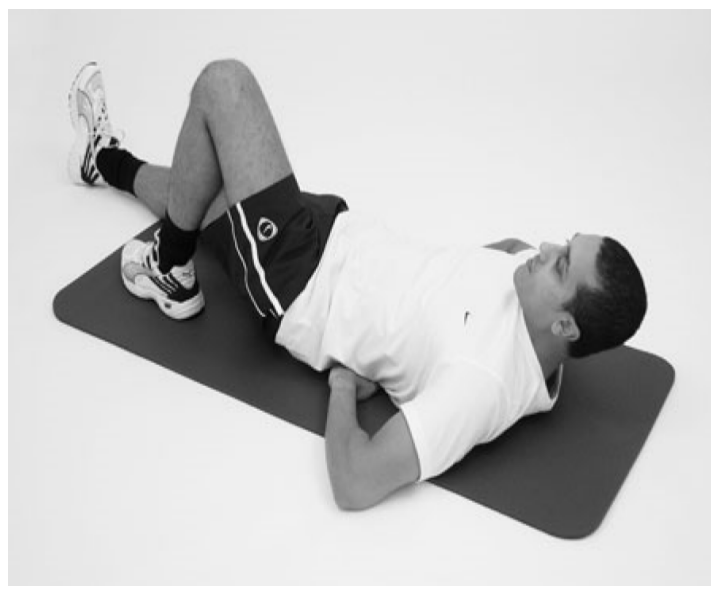

(b)

JMR

Figure 7. Seventh exercise: Rising the head and shoulders

distribution of data. Because of the unequal groups (control group, $n=12$ ), we used Scheffe post hoc test.

\section{Results}

\section{Descriptive statistics}

In Table 1, the demographic characteristics of study participants such as height, weight, work experience and age, were presented using the minimum, maximum, mean, and standard deviation in 3 groups of electrotherapy, core stability exercises, and control. One-way analysis of variance showed no significant difference between mean height $\left(\mathrm{P}=0.183, \mathrm{~F}_{2,39}=1.177\right)$, mean weight $(\mathrm{P}=0.180$, $\left.\mathrm{F}_{2,39}=1.79\right)$, mean experience background $(\mathrm{P}=0.151$, $\left.\mathrm{F}_{2,39}=1.098\right)$, and mean age $\left(\mathrm{F}_{2,39}=2.04, \mathrm{P}=0.143\right)$ of mother assistants working in disabled children care units in all groups of core stability exercises, electrotherapy and control. Thus, the three groups are matched with respect to age, height, weight, and work experience.

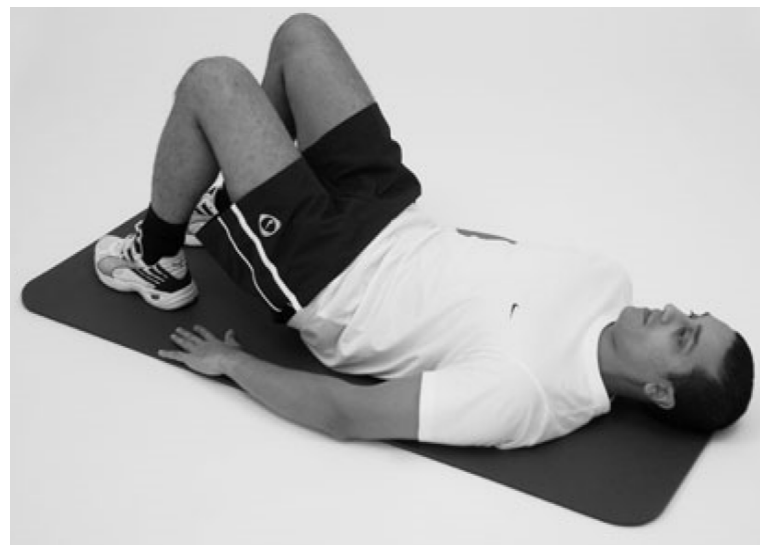

(a)

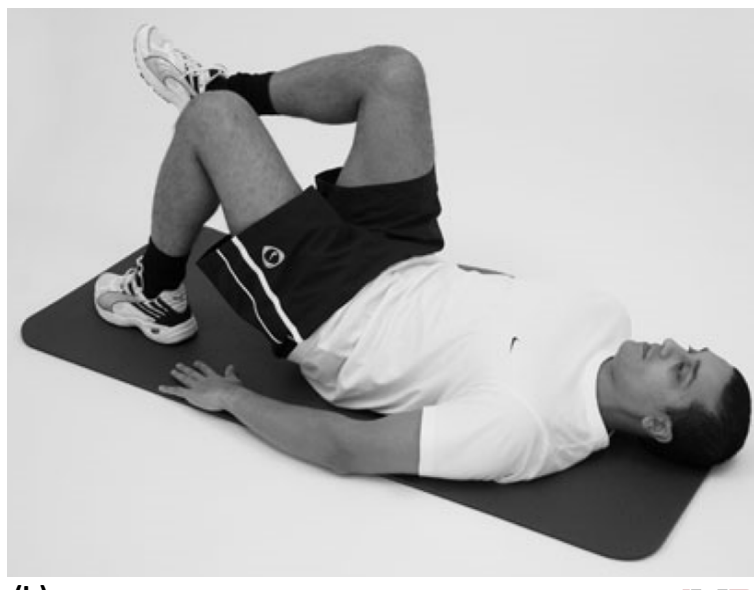

(b)

JMR

Figure 8. Eighth exercise: Muscle contraction of the lower abdomen

Table 2, show average and the standard deviation of research variables as well as the amount of the chronic LBP in the pretest and posttest based on the difference between pre-and post-test scores of two groups of core stability exercises and electrotherapy.

\section{Assumption of normal distribution of data}

The results of Shapiro-Wilk test scores in Table 3 show that the the score differences between the pretest and posttests of reasearch variables are normally distributed $(\mathrm{P}<0.05)$. The 1 -way ANOVA results in Table 4 indicate significant differences with respect to mean scores of assistants' chronic LBP, among groups of core stability exercises (-24.4), electrotherapy (31.5) and control (1.7). $\left(\mathrm{F}_{2.39}=56.1, \mathrm{P}=0.000\right)$. Therefore, the Scheffe post hoc test was used to test the study hypothesis and determine the source of the differences.

The Scheffe post hoc test results revealed a significant difference between the electrotherapy group (-31.5), and control $(-1.7)(\mathrm{P}=0.000)$ with regard to the mean scores 
Table 1. The demographic variables

\begin{tabular}{|c|c|c|c|c|c|}
\hline Groups & Variable & Min & Max & Mean & SD \\
\hline \multirow{4}{*}{ Electrotherapy } & Height (cm) & 150 & 170 & 159.06 & 6.2 \\
\hline & Weight (kg) & 46 & 86 & 6.66 & 11.6 \\
\hline & Work experience (y) & 7 & 31 & 18.9 & 8.3 \\
\hline & Age (y) & 30 & 57 & 43 & 8.6 \\
\hline \multirow{4}{*}{$\begin{array}{c}\text { Core stability } \\
\text { exercises }\end{array}$} & Height (cm) & 155 & 172 & 162.9 & 5.5 \\
\hline & Weight (kg) & 59 & 98 & 75.4 & 13.1 \\
\hline & Work experience (y) & 5 & 30 & 14.3 & 8.4 \\
\hline & Age (y) & 32 & 55 & 40.5 & 8.9 \\
\hline \multirow{4}{*}{ Control } & Height (cm) & 150 & 174 & 162.7 & 6.9 \\
\hline & Weight (kg) & 54 & 76 & 65.9 & 6.5 \\
\hline & Work experience (y) & 10 & 22 & 13.8 & 4.3 \\
\hline & Age (y) & 30 & 45 & 36.7 & 35 \\
\hline \multirow{4}{*}{ Total } & Height (cm) & 150 & 174 & 161.5 & 6.3 \\
\hline & Weight (kg) & 46 & 98 & 69 & 11.8 \\
\hline & Work experience (v) & 5 & 31 & 158 & 76 \\
\hline & Age $(y)$ & 30 & 57 & 40.3 & 8.1 \\
\hline
\end{tabular}

of working mother assistants' chronic LBP. Therefore, the null hypothesis was rejected. In other words, electrotherapy significantly improved chronic non-specific LBP in mother assistants.

Scheffe post hoc test results showed a significant difference between electrotherapy group (-31.5) and core stability exercise group $(-24.4)(\mathrm{P}=0.046)$ with respect to the mean scores of working mother assistants' chronic LBP. Therefore, the null hypothesis was rejected. In other words, a course of electrotherapy compared to core stability exercises significantly reduced chronic non-specific LBP in mother assistants. Meanwhile, the results of dependent $t$ test to assess changes within groups showed that electrotherapy brought about a significant difference between the mean scores of mother assistants' nonspecific chronic LBP from pretest to posttest ( $\mathrm{t} 14=13.5$, $\mathrm{P}=0.000$ ) The core stabilization exercises caused a significant difference in the mean scores of mother assistants' non-specific chronic LBP from pretest to posttest $(\mathrm{t} 14=11.43, \mathrm{P}=0.000)$. In the control group, chronic non-

Table 2 . The values of research variables

\begin{tabular}{cccccccc}
\hline \multirow{2}{*}{ Variable } & Groups & \multicolumn{2}{c}{ Pretest } & \multicolumn{2}{c}{ Posttest } & \multicolumn{2}{c}{ Pretest Difference Scores } \\
\cline { 3 - 8 } & & Mean & SD & Mean & SD & Mean & SD \\
\hline \multirow{2}{*}{$\begin{array}{c}\text { Chronic } \\
\text { low back } \\
\text { pain }\end{array}$} & Electrotherapy & 46.5 & 12.05 & 15 & 7.8 & -31.5 & 9.02 \\
& Core stability & 36.5 & 9.5 & 12.06 & 7.2 & -24.4 & 8.3 \\
& Control & 44.1 & 10.1 & 42.4 & 9.8 & -1.75 & 2.4 \\
\hline
\end{tabular}


Table 3. Results of Shapiro-Wilk test

\begin{tabular}{|c|c|c|c|c|}
\hline Variable & Groups & D & df & Sig. \\
\hline \multirow{3}{*}{ Chronic low back pain } & Electrotherapy & 0.969 & 15 & 0.849 \\
\hline & Core stability & 0.943 & 15 & 0.415 \\
\hline & Control & 0.905 & 12 & 0.182 \\
\hline
\end{tabular}

Table 4. The results of ANOVA test

\begin{tabular}{cccccc}
\hline Source & SS & df & MS & F & Sig. \\
\hline Intergroup & 6280.8 & 2 & 3140.3 & & \\
Intergroup & 2181.7 & 39 & 55.9 & & 0.000 \\
Total & 8462.5 & 41 & & & \\
\hline
\end{tabular}

specific LBP in mother assistants significantly reduced from pretest to posttest $(\mathrm{t} 11=2.43, \mathrm{P}=0.033)$.

\section{Discussion}

In patients with chronic LBP, the control ability of trunk muscles is impaired and the activity of deep muscles decreases [23]. These are muscles that support posture against gravity probably due to the inhibition of pain reflex, delays in activity, decreased tone, lower muscle power and tolerance followed by stiffness of the ligament.

Muscular endurance is an important factor in patients with LBP. Moreover, trunk muscles in almost all activities, whether sitting, standing, or even falling are active and their weakness affects the pattern of motion and subsequently causes pain. Therefore, these muscles must be active all day long. Lack of endurance in these muscles can be an important factor in motor impairment and LBP. Core stability exercise reduces pain in such patients by increasing muscular endurance [5]. These exercises influence the pain perception and evaluation abilities.

Local stabilizers are small deep muscles of the spine that bind the various pieces together and stabilize them. They include the multifidus muscle, the transverse abdominis and oblique abdominal. Moreover, large muscles of the rectus abdominis and lumbar muscles are responsible for producing movements of the lumbar area7. Core stabilization exercises can reverse type II muscle atrophy in the multifidus muscle in back and by increasing the diameter of the muscles alleviate pain in patients7. By inceasing trunk stability, additional forces damaging the spine are reduced and pain gets better. Increasing stability, improves the natural alignment of the spine and movement patterns and reduces harmful passive pressures that hold spine [24]. Because core stability exercises increase endurance and strengthen multifidus muscle and the transvers abdominal, it seems that the cause of pain in mother assistants after the intervention can be justified.

Scheffe post hoc test results showed that a course of electrotherapy compared to core stability exercises led to a significant reduction in nonspecific chronic LBP. The result of comparing the effects of stabilization exercise and electrotherapy is inconsistent with the results of some other studies [15], including Kumar Saravana [24]. The positive performance of the core muscles decreases too much pressure and reduces or eradicates the perception of pain. Basically, the purpose of provocation, is creating signals coming from the environment to alter the excitability of the central components of the neurological system, and changing sensory input alters perception of pain signals. These stimulations are unable to change the primary cause of pain but they change the feeling and perception of pain signals [15].

There is a morphine-like effect on pain control by using $\mathrm{C}$ fibers. In this system, enkephalin secretion by interstitial neurons in the dorsal horn of spinal cord activated by stimulating A gamma pain receptor fiber inhibits pain. 